\title{
Stable Fluorescent and Enzymatic Tagging of Bradyrhizobium diazoefficiens to Analyze Host-Plant Infection and Colonization
}

\author{
Raphael Ledermann, Ilka Bartsch, Mitja N. Remus-Emsermann, Julia A. Vorholt, \\ and Hans-Martin Fischer \\ ETH Zurich, Institute of Microbiology, Vladimir-Prelog-Weg 4, CH-8093 Zurich, Switzerland \\ Submitted 3 March 2015. Accepted 21 May 2015.
}

\begin{abstract}
Bradyrhizobium diazoefficiens USDA 110 (formerly named Bradyrhizobium japonicum) can fix dinitrogen when living as an endosymbiont in root nodules of soybean and some other legumes. Formation of a functional symbiosis relies on a defined developmental program mediated by controlled gene expression in both symbiotic partners. In contrast to other well-studied Rhizobium-legume model systems that have been thoroughly examined by means of genetically tagged strains, analysis of $\boldsymbol{B}$. diazoefficiens host infection has been impaired due to the lack of suitable tagging systems. Here, we describe the construction of $B$. diazoefficiens strains constitutively expressing single-copy genes for fluorescent proteins (eBFP2, mTurquoise2, GFP+, sYFP2, mCherry, HcRed) and enzymes (GusA, LacZ). For stable inheritance, the constructs were recombined into the chromosome. Effectiveness and versatility of the tagged strains was demonstrated in plant infection assays. (i) The infection process was followed from root-hair attachment to colonization of nodule cells with epifluorescent microscopy. (ii) Monitoring mixed infections with two strains producing different fluorescent proteins allowed rapid analysis of nodule occupancy and revealed that the majority of nodules contained clonal populations. (iii) Microscopic analysis of nodules induced by fluorescent strains provided evidence for host-dependent control of $B$. diazoefficiens bacteroid morphology in nodules of Aeschynomene afraspera and Arachis hypogaea (peanut), as deduced from their altered morphology compared with bacteroids in soybean nodules.
\end{abstract}

Formation of the intimate symbiosis between rhizobia and their legume host plants follows a complex, strictly controlled developmental program that eventually leads to the massive colonization of specialized plant organs, root nodules, by

Current address for M. N. Remus-Emsermann: Federal Department of Economic Affairs, Education and Research EAER, Agroscope, Institute for Food Sciences IFS, Schloss 1, P.B., 8820 Wädenswil, Switzerland

Corresponding author: H.-M. Fischer: Telephone: +41 4463244 19; Fax: +4144632 13 78; E-mail: fischeha@ethz.ch

*The $\boldsymbol{e}$-Xtra logo stands for "electronic extra" and indicates that three supplementary figures, two supplementary tables, and supplementary methods are published online.

(c) 2015 The American Phytopathological Society nitrogen-fixing endosymbionts (Downie 2014; Oldroyd et al. 2011). A number of model systems have been studied intensively in the past at the cellular, biochemical, and molecular genetic levels of both symbiotic partners. The analysis of infection and colonization processes was greatly stimulated by the use of light microscopy in combination with genetically tagged rhizobial strains that express fluorescent or enzymatic proteins (Gage 2002; Oldroyd and Downie 2008). While such tagged rhizobial strains are well-established for model systems like Sinorhizobium-Medicago or Mesorhizobium-Lotus and others (Stuurman et al. 2000), similar tools are largely lacking for the soybean symbiont Bradyrhizobium diazoefficiens USDA 110 (formerly named Bradyrhizobium japonicum) (Delamuta et al. 2013) despite the agricultural significance of and scientific interest in the latter system. In previous investigations of the $B$. diazoefficiens-soybean symbiosis, staining techniques and electron microscopy were used to visualize the endosymbionts at different stages of the infection process (Pueppke 1983; Turgeon and Bauer 1985). Lack of endogenous plasmids in $B$. diazoefficiens and the rapid loss even of broad-host range plasmids in the absence of selective pressure (Avarez-Morales et al. 1986; Stuurman et al. 2000) have hampered the development of versatile plasmid-based tagging tools that are widely used in other systems. Chromosomal integration of respective marker genes offers an alternative for stable genetic tagging. In fact, transposons have been used to integrate genes for fluorescent proteins in different alphaproteobacteria, including Bradyrhizobium strains (Bhatia et al. 2002; Hayashi et al. 2014; Matthysse et al. 1996; Okubo et al. 2013; Schada von Borzyskowski et al. 2015). However, this strategy yielded random chromosomal insertions that may have undesired secondary effects, depending on the location of the inserted transposon. To circumvent this limitation, Tn7-based marker-delivery systems were developed that enabled site-specific insertion of markers at a single att $\operatorname{Tn} 7$ site present in different gram-negative bacteria (Choi et al. 2005; Koch et al. 2001). However, since this system turned out to work inefficiently in B. diazoefficiens (our own unpublished results), we have designed an integrative vector serving as a basis for stable tagging of $B$. diazoefficiens with either one of six fluorescent or two enzymatic proteins. The respective nonreplicative plasmids were introduced by conjugation into $B$. diazoefficiens and were chromosomally integrated via homologous recombination in a symbiotically dispensable region. Appropriate transcription of the singlecopy marker genes was ensured by the use of strong constitutive promoters. Here, we describe the construction of this novel 
genetic toolset for $B$. diazoefficiens and demonstrate its utility for the cellular analysis of developmental steps during formation of the symbiotic interaction between $B$. diazoefficiens and host plants. In another application, we used differently tagged strains for mixed infection assays and assessed nodule occupancy based on the distinct fluorescent properties of the inoculated strains.

\section{RESULTS AND DISCUSSION}

\section{Chromosomal integration of single-copy genes encoding fluorescent or enzymatic proteins in B. diazoefficiens.}

We constructed 13 derivatives of $B$. diazoefficiens $110 s p c 4$, which constitutively express fluorescent (11) or enzymatic (2) protein tags from single-copy genes integrated in the chromosome (Table 1). Chromosomal integration was crucial because of the notorious tendency of $B$. diazoefficiens to loose plasmids in the absence of antibiotic selection, e.g., in root nodules. All constructs were chromosomally integrated at the same symbiotically dispensable region downstream of the scoI gene (Bühler et al. 2010). Comparative soybean infection tests with tagged strains and untagged wild type revealed that integration of plasmids at this locus affected neither symbiotic properties nor nodulation competitiveness (Supplementary Fig. S1). Two different, constitutive promoters (Paph originating from the kanamycin-resistance gene aphII of Tn 5 and PtuF of the gene for elongation factor thermo-unstable ( $\mathrm{Tu})$ of Methylobacterium extorquens AM1) were tested for driving transcription of four of the seven genes encoding fluorescent proteins. Because all genes were present in single copy only, the rather strong Paph and PtuF promoters were chosen to ensure appropriate fluorescence intensities. The latter promoter was characterized previously and was shown to function efficiently in B. diazoefficiens (Schada von Borzyskowski et al. 2015). While our previously generated transcriptomics data suggested that the promoter of the B. diazoefficiens rrn operon is probably even stronger (Hauser et al. 2007; Pessi et al. 2007), we refrained from using it here because very high levels of tagging proteins may result in undesired secondary effects.

Comparison of fluorescence intensity derived from mCherry expressed from Paph or PtuF revealed no significant differences between the two promoters (data not shown). Different fluorophores, however, exhibited different fluorescence intensities in B. diazoefficiens (Fig. 1A). Because fluorescence of B. diazoefficiens GFP-1 was rather weak, the codon composition of the respective GFP+ (green fluorescent protein) gene was optimized according to the codon frequency of B. diazoefficiens (Grote et al. 2005; Ramseier and Göttfert 1991). The resulting synthetic bjGFP gene (Supplementary Fig. S2) was placed under the control of Paph and was integrated into the chromosome to yield strain bjGFP-1. Cells of this strain exhibited a drastically increased fluorescence compared with strain GFP-1 (data not shown).

Based on fluorescence intensity, photobleaching susceptibility, and plant tissue autofluorescence, we found strains expressing mCherry (mChe-1, mChe-4), sYFP2 (sYFP2-1, sYFP-4), or $b j G F P$ (bjGFP-1) the most useful. However, for visualization of $B$. diazoefficiens cells in plant tissue that contains plastids exhibiting red autofluorescence (e.g., stem nodules of Aeschynomene afraspera), the use of blue- or cyantagged (eBFP2-1, eBFP2-4 or mTq2-1, mTq2-4, respectively) bacteria may be superior, with eBFP2 being less favorable because of its pronounced susceptibility to photobleaching

To characterize the excitation and emission spectra of the constructed $B$. diazoefficiens strains, spectra of respective cell suspensions were recorded (Fig. 1B). The excitation and emission maxima were as follows (in $\mathrm{nm}$ ): $385 / 450$ (strain
eBFP2-1), 456/474 (mTq2-1), 497/509 (bjGFP-1), 505/540 (sYFP2-1), 590/606 (mChe-1), and 591/635 (HcRed-1). When signal intensities of the emission maxima were normalized to the number of cells present in the suspensions, the following order in fluorescence intensity was found: sYFP2-1 > mTq2-1 > eBFP2-1 > bjGFP-1 > mChe-1 > HcRed-1 (Fig. 1A).

Notably, the excitation spectrum of $\mathrm{mTq} 2-1$ cells exhibited two local maxima (435 and $456 \mathrm{~nm}$ ), as described also for the purified protein (Goedhart et al. 2012). Unlike the purified protein, however, excitation absorption of mTq2-1-tagged cells was more pronounced at 456 than at $435 \mathrm{~nm}$ in B. diazoefficiens. Similarly, HcRed-1 cells had two emission peaks, with the more pronounced one at $635 \mathrm{~nm}$ blue-shifted by $5 \mathrm{~nm}$, as compared with the purified HcRed protein (Gurskaya et al. 2001) and a minor one around $618 \mathrm{~nm}$, which was specified as maximum for HcRed in another study (Day and Davidson 2009). Minor differences between the in vitro and in vivo spectral properties of fluorescent proteins are not uncommon and are likely due to different physical and chemical conditions prevailing in solution and in cells (Mylle et al. 2013). For example, the presence of pigments or other chromophores in particular cells may affect the spectral features of fluorescent proteins.

\section{Visualization of early infection stages.}

Tagged B. diazoefficiens strains were used to visualize roothair attachment and early stages of soybean infection. Strains mChe-1 (or mChe-4 in some experiments), bjGFP-1, and GusA-1 were inoculated on soybean seedlings that were then incubated for 4 days, before epidermal cell layers of roots were peeled off and inspected by epifluorescence microscopy or stained with X-gluc (5-bromo-4-chloro-3-indolyl- $\beta$-D-glucuronic acid). Individual fluorescent bacteria were readily detectable, mostly being attached in a polar manner (Fig. 2A). Colonization of roots was not uniform but was most pronounced in zones with dense root hairs, whereas only a few bacteria were attached on epidermal cells (not shown).

Soybean seedlings are infected by $B$. diazoefficiens via thin infection threads emerging from microcolonies entrapped between short deformed root hairs and epidermal cells (Pueppke 1983; Turgeon and Bauer 1985). With the help of tagged strains, bacteria-containing infection threads could be observed in great detail. Soon after penetration of the root hair, infection threads began to branch leading to several threads within a single root-hair cell (Fig. 2B). Given the thin nature of soybean infection threads, invading bacteria were lined up in a file of individual cells (Fig. 2C).

Infection threads in adjacent root-hair cells were mostly populated with only one type of bacterium when GFP- and mCherry-tagged strains were inoculated simultaneously (Fig. 2D). However, in rare cases when entrapped microcolonies consisted of mixed populations, we also observed root hairs with multiple infection threads harboring either strain (Fig. 2E). Notably, we never detected individual infection threads harboring more than one strain while analyzing approximately 50 infection threads on each of two soybean plants. This is different from what has been described for Medicago sativa and vetch, in which different bacterial strains of Sinorhizobium meliloti and Rhizobium leguminosarum bv. viciae, respectively, coexisted in a single infection thread, which may be related to the thicker nature of infection threads in those hosts (Gage 2002; Gage et al. 1996; Stuurman et al. 2000). While infection threads of Medicago sativa ramify only after reaching the cortical cell layers, soybean root-hair cells frequently showed multiple infection threads that may have originated from early branching or multiple, independently formed threads. The latter situation may explain the formation of mixed infected nodules described below. 
An application of GusA-tagged bacteria is illustrated in Figure 2F, showing an overview of a longitudinal section that was cut from a soybean root 4 days after inoculation with B. diazoefficiens GusA-1. Localization of emerging nodule primordia that are characterized by small, anticlinal dividing cells is facilitated by the dark precipitated product resulting from X-gluc hydrolysis by the GusA-tagged bacteria present in infection threads.
Use of tagged strains to study nodule occupancy.

We have used $B$. diazoefficiens strains mChe-4 and sYFP2-4 in mixed-infection experiments to study colonization of soybean, cowpea, mung bean, and siratro nodules. These strains, which express the respective fluorescent proteins from the PtuF promoter, were readily detected in infected host cells (Fig. 3). This documents that the PtuF promoter is equally as well-suited as the Paph promoter to drive transcription of the genes encoding

Table 1. Bacterial strains and plasmids

\begin{tabular}{|c|c|c|}
\hline Strain or plasmid & Relevant phenotype or genotype $^{a}$ & Source or reference \\
\hline \multicolumn{3}{|l|}{ Escherichia coli } \\
\hline DH5 $\alpha$ & $\begin{array}{l}\text { supE44 } \Delta \text { lacU169 ( } \varphi 80 \text { lacZUM15) hsdR17 } \\
\text { recA1 gyrA96 thi-1 relA2 }\end{array}$ & BRL, Gaithersburg, MD, U.S.A. \\
\hline S17-1 $\lambda$ pir & $\begin{array}{l}\mathrm{Sm}^{\mathrm{r}} \mathrm{Sp}^{\mathrm{r}} h s d R \text { (RP4-2 kan::Tn7 tet }:: M u \\
\text { chromosomal integrated) }\end{array}$ & de Lorenzo et al. 1993 \\
\hline \multicolumn{3}{|c|}{ Bradyrhizobium diazoefficiens } \\
\hline $110 s p c 4$ & $\mathrm{Sp}^{\mathrm{r}}$ wild type (USDA 110 derivative) & Regensburger and Hennecke 1983 \\
\hline eBFP2-1 & $\begin{array}{l}\mathrm{Sp}^{\mathrm{r}} \mathrm{Tc}^{\mathrm{r}} 110 s p c 4 \text { with chromosomal } \\
\text { integration of pRJPaph-eBFP2 }\end{array}$ & This work \\
\hline $\mathrm{mTq} 2-1$ & $\begin{array}{l}\mathrm{Sp}^{\mathrm{r}} \mathrm{Tc}^{\mathrm{r}} 110 s p c 4 \text { with chromosomal } \\
\text { integration of pRJPaph-mTq2 }\end{array}$ & This work \\
\hline GFP-1 & $\begin{array}{l}\mathrm{Sp}^{\mathrm{r}} \mathrm{Tc}^{\mathrm{r}} \text { 110spc4 with chromosomal } \\
\text { integration of pRJPaph-GFP }\end{array}$ & This work \\
\hline bjGFP-1 & $\begin{array}{l}\mathrm{Sp}^{\mathrm{r}} \mathrm{Tc}^{\mathrm{r}} 110 s p c 4 \text { with chromosomal } \\
\text { integration of pRJPaph-bjGFP }\end{array}$ & This work \\
\hline sYFP2-1 & $\begin{array}{l}\mathrm{Sp}^{\mathrm{r}} \mathrm{Tc}^{\mathrm{r}} 110 s p c 4 \text { with chromosomal } \\
\text { integration of pRJPaph-sYFP2 }\end{array}$ & This work \\
\hline mChe-1 & $\begin{array}{l}\mathrm{Sp}^{\mathrm{r}} \mathrm{Tc}^{\mathrm{r}} 110 s p c 4 \text { with chromosomal } \\
\text { integration of } \mathrm{pRJPaph}-\mathrm{mChe}\end{array}$ & This work \\
\hline HcRed-1 & $\begin{array}{l}\mathrm{Sp}^{\mathrm{r}} \mathrm{Tc}^{\mathrm{r}} 110 s p c 4 \text { with chromosomal } \\
\text { integration of pRJPaph-HcRed }\end{array}$ & This work \\
\hline eBFP2-4 & $\begin{array}{l}\mathrm{Sp}^{\mathrm{r}} \mathrm{Tc}^{\mathrm{r}} 110 s p c 4 \text { with chromosomal } \\
\text { integration of pRJPtuF-eBFP2 }\end{array}$ & This work \\
\hline $\mathrm{mTq} 2-4$ & $\begin{array}{l}\mathrm{Sp}^{\mathrm{r}} \mathrm{Tc}^{\mathrm{r}} 110 s p c 4 \text { with chromosomal } \\
\text { integration of pRJPtuF-mTq2 }\end{array}$ & This work \\
\hline sYFP2-4 & $\begin{array}{l}\mathrm{Sp}^{\mathrm{r}} \mathrm{Tc}^{\mathrm{r}} 110 s p c 4 \text { with chromosomal } \\
\text { integration of pRJPtuF-sYFP2 }\end{array}$ & This work \\
\hline mChe-4 & $\begin{array}{l}\mathrm{Sp}^{\mathrm{r}} \mathrm{Tc}^{\mathrm{r}} 110 s p c 4 \text { with chromosomal } \\
\text { integration of pRJPtuF-mChe }\end{array}$ & This work \\
\hline GusA-1 & $\begin{array}{l}\mathrm{Sp}^{\mathrm{r}} \mathrm{Tc}^{\mathrm{r}} 110 s p c 4 \text { with chromosomal } \\
\text { integration of pRJPaph-gusA }\end{array}$ & This work \\
\hline LacZYA-1 & $\begin{array}{l}\mathrm{Sp}^{\mathrm{r}} \mathrm{Tc}^{\mathrm{r}} 110 s p c 4 \text { with chromosomal } \\
\text { integration of pRJPaph-lacZYA }\end{array}$ & This work \\
\hline \multicolumn{3}{|c|}{ 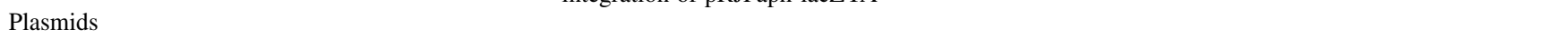 } \\
\hline pRJPaph-gfp & $\begin{array}{l}\mathrm{Tc}^{\mathrm{r}}(\mathrm{pRJ} 2575) \text { Paph- } g f p+\text { for integration } \\
\text { downstream of } s c o I\end{array}$ & This work \\
\hline pRJPaph-gfp_a1 & $\begin{array}{l}\mathrm{Tc}^{\mathrm{r}} \text { (pRJPaph-gfp) replaced PstI site with } \\
\text { AatII and KpnI sites }\end{array}$ & This work \\
\hline pRJPaph-eBFP2 ${ }^{b}$ & $\begin{array}{l}\mathrm{Tc}^{\mathrm{r}} \text { (pRJPaph-gfp_a1) Paph-eBFP2 for } \\
\text { integration downstream of scoI }\end{array}$ & This work \\
\hline pRJPaph-mTq $2^{\mathrm{b}}$ & $\begin{array}{l}\mathrm{Tc}^{\mathrm{r}} \text { (pRJPaph-gfp_a1) Paph-mTurquoise } 2 \text { for } \\
\text { integration downstream of scoI }\end{array}$ & This work \\
\hline pRJPaph-bjGFP & $\begin{array}{l}\mathrm{Tc}^{\mathrm{r}} \text { (pRJPaph-gfp_a1) Paph-bjGFP for } \\
\text { integration downstream of scoI }\end{array}$ & This work \\
\hline pRJPaph-sYFP2 ${ }^{\mathrm{b}}$ & $\begin{array}{l}\mathrm{Tc}^{\mathrm{r}} \text { (pRJPaph-gfp_a1) Paph-sYFP2 for } \\
\text { integration downstream of scoI }\end{array}$ & This work \\
\hline pRJPaph-mChe ${ }^{b}$ & $\begin{array}{l}\mathrm{Tc}^{\mathrm{r}} \text { (pRJPaph-gfp_a1) Paph- } m \text { Cherry for } \\
\text { integration downstream of scoI }\end{array}$ & This work \\
\hline pRJPaph-HcRed ${ }^{b}$ & $\begin{array}{l}\mathrm{Tc}^{\mathrm{r}}(\mathrm{pRJPaph}-\mathrm{gfp}) \text { Paph-HcRed for } \\
\text { integration downstream of scoI }\end{array}$ & This work \\
\hline pRJPtuF-eBFP2 & $\begin{array}{l}\mathrm{Tc}^{\mathrm{r}} \text { (pRJPaph-gfp_a1) PtuF-eBFP2 for } \\
\text { integration downstream of scoI }\end{array}$ & This work \\
\hline pRJPtuF-mTq2 & $\begin{array}{l}\mathrm{Tc}^{\mathrm{r}} \text { (pRJPaph-gfp_a1) PtuF-mTurquoise } 2 \text { for } \\
\text { integration downstream of scoI }\end{array}$ & This work \\
\hline pRJPtuF-sYFP2 & $\begin{array}{l}\mathrm{Tc}^{\mathrm{r}} \text { (pRJPaph-gfp_a1) PtuF-sYFP2 for } \\
\text { integration downstream of scoI }\end{array}$ & This work \\
\hline pRJPtuF-mChe & $\begin{array}{l}\mathrm{Tc}^{\mathrm{r}} \text { (pRJPaph-gfp_a1) PtuF-mCherry for } \\
\text { integration downstream of scoI }\end{array}$ & This work \\
\hline pRJPaph-gusA ${ }^{\mathrm{b}}$ & $\begin{array}{l}\mathrm{Tc}^{\mathrm{r}} \text { (pRJPaph-gfp_a1) Paph-gusA for } \\
\text { integration downstream of scoI }\end{array}$ & This work \\
\hline pRJPaph-lacZYA & $\begin{array}{l}\mathrm{Tc}^{\mathrm{r}} \text { (pRJPaph-gfp) Paph-lacZYA for } \\
\text { integration downstream of scoI }\end{array}$ & This work \\
\hline
\end{tabular}

${ }^{\mathrm{a}} \mathrm{Sm}^{\mathrm{r}}, \mathrm{Sp}^{\mathrm{r}}, \mathrm{Tc}^{\mathrm{r}}=$ streptomycin-, spectinomycin-, and tetracycline-resistant.

$\mathrm{b}$ Deposited in the Addgene plasmid repository. 
fluorescent tags. Based on epifluorescence microscopy, four different colonization patterns could be distinguished. The vast majority of all inspected nodules were colonized by only one of the two strains, supporting the idea that rhizobial populations in legume nodules are largely clonal (Pobigaylo et al. 2008; Simms et al. 2006) (Figs. 3A and 4). In some of the analyzed nodules, however, both strains were present. The two populations were either separated by uninfected cells, suggesting that these nodules originated from two independent but spatially close infection events ('fused nodules') (Fig. 3B) or present in immediately adjacent host cells ('mixed nodules') (Fig. 3C). Mixed nodules might be the result of simultaneous entry of both strains present in branched infection threads in individual root-hair cells (Fig. 2E). At a very low frequency, individual host cells harboring both symbionts were detected in a few nodules (Fig. 3D, arrowheads). Based on the results described above, we assume that the different symbionts entered these host cells via distinct infection threads.

Enzymatically tagged strains GusA-1 and LacZYA-1 turned out to be very useful for obtaining a rapid overview of nodule occupancy (Fig. 3E and F). In a mixed infection experiment with these strains, nodules populated by one or both strains were readily distinguished by simultaneous staining with X-gluc and green- $\beta$-D-gal (1-methyl-3-indolyl- $\beta$-D-galactopyranoside).

We also performed quantitative mixed infection tests with different hosts, including three soybean varieties ('Green Butterbean', Williams 82, 'Black Jet') and three additional legume species, Vigna radiata (mungbean), Vigna unguiculata (cowpea), and Macroptilium atropurpureum (siratro). For inoculation, 1:1 or 10:1 mixtures of $B$. diazoefficiens strains expressing sYFP2 or mCherry containing a constant total number of $10^{3}$ cells (Fig. 4A and B) or 1:1 mixtures containing $10^{2}, 10^{4}, 10^{6}, 10^{8}$, or $10^{10}$ total cells (Fig. 4C) were used. As described above for soybean cv. Green Butterbean, the great majority of the nodules were colonized by only one strain, regardless of the host plant, and only a few fused or mixed nodules were detected (Fig. 4A). The ratio of sYFP2-4 to mChe-4-only populated nodules reflected the ratio of the strains in the inoculum, i.e., about 1:1 and 10:1 in the experiments shown in Figure 4A and B. Increasing cell numbers in 1:1 inoculum mixtures revealed no differences with regard to the macroscopic plant phenotype and total number of nodules, regardless of the inoculum size (data not shown). Moreover, the ratio between different nodules types was roughly stable when $10^{2}, 10^{4}, 10^{6}$, or $10^{8}$ cells were inoculated per plant (Fig. $4 \mathrm{C}$ ). Only when as many as $10^{10}$ bacteria were applied per seedling was a slight increase of fused nodules observed, yet even under these high-density inoculation conditions more than $50 \%$ of all nodules were populated with only one strain.

Fluorescently tagged rhizobia have been used previously to study formation and colonization of mixed infected nodules. Examples include the Sinorhizobium meliloti-Medicago sativa or Bradyrhizobium sp. strain ORS285-Aschynomene spp. symbioses, which differ in the mode of host entry (infection thread-dependent versus crack entry) and the type of nodules (indeterminate versus determinate) (Bonaldi et al. 2011; Gage 2002). To our knowledge, this is the first study in which fluorescently tagged $B$. diazoefficiens strains were used to follow early infection stages and colonization of soybeans and related legumes, which are all infected via threads to develop determinate nodules. A notable result of the mixed infection experiments was our observation that the majority of nodules contained clonal populations, even when a very dense inoculum was applied. This finding combined with the high spatial density of primary infection sites and emerging nodule primordia (Fig. 2F) suggests that a large proportion of primary infection events are abortive, which is in agreement with similar observations made previously by Calvert et al. (1984).

\section{B. diazoefficiens bacteroid morphology is host-dependent.}

For indeterminate symbioses, it is well established that bacteroid differentiation, including morphological alterations, is dependent on the host plant (Kondorosi et al. 2013; Mergaert et al. 2006; Oono et al. 2010). It was previously shown for the photosynthetic Bradyrhizobium sp. strain ORS285 that bacteroids of determinate Aeschynomene legume nodules may either be rod shaped (though enlarged) in A. afraspera but spherical in A. indica (Bonaldi et al. 2011). The most prominent host of $B$. diazoefficiens is soybean, which belongs to the Millettioid clade of legumes and forms determinate nodules containing rod-shaped bacteroids that are morphologically very similar to free-living cells. In addition to soybean, B. diazoefficiens is able to induce determinate nodules on several other host plants,
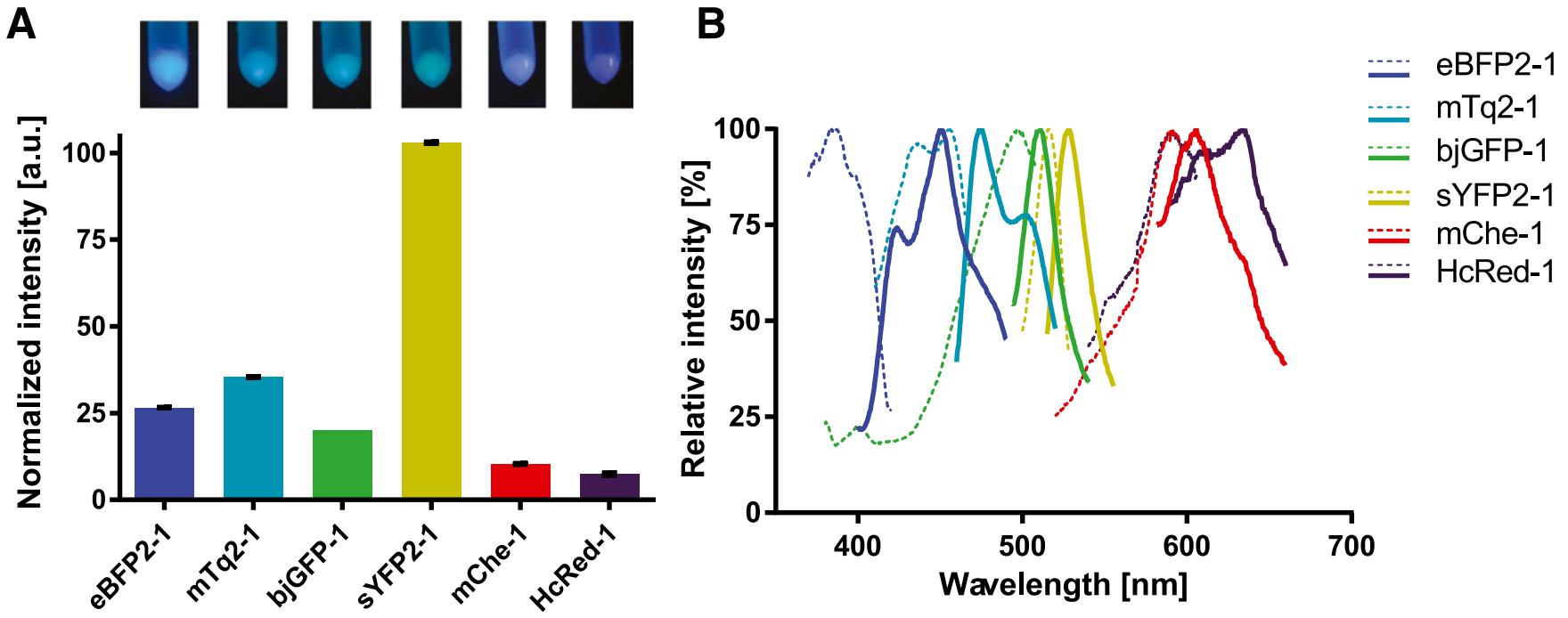

Fig. 1. Fluorescence of free-living Bradyrhizobium diazoefficiens cells harboring chromosomally integrated genes that encode different fluorescent proteins. A, Fluorescence of cell sediments irradiated with a 365-nm light source (above) and maximal fluorescence intensity of cell suspension (arbitrary units) deduced from the emission spectra shown in panel B and normalized to the CFU counts of the respective cell suspension (below). B, Excitation (dotted lines) and emission spectra (solid lines) of the indicated strains (optical density at $600 \mathrm{~nm}=0.75$ for all strains except $\mathrm{s} Y$ FP2-1, in which the respective value was 0.15 ). Relative fluorescent intensities are shown with the maximum of each individual spectrum defined as $100 \%$. 
including V. radiata, V. unguiculata, Macroptilium artropurpureum, A. afraspera, and Arachis hypogaea (peanut) (Göttfert et al. 1990a; Noisangiam et al. 2012; Renier et al. 2011). Yet, little is known about the morphology of $B$. diazoefficiens bacteroids in the latter two species, which belong to the Dalbergioid clade of legumes. We used mCherry-tagged B. diazoefficiens in combination with confocal fluorescence microscopy to compare the morphology of bacteroids present in nodules of soybean, A. afraspera, and peanut (Fig. 5). While bacteroids in soybean nodules were small and rod-shaped like free-living cells (Fig. 5A), we found that bacteroids in nodules of peanut and A. afraspera underwent morphological changes. While bacteroids in A. afraspera nodules retained a rod-like morphology, most of them were enlarged to varying extents (Fig. 5B). Most strikingly, the development of bacteroids in peanut nodules was accompanied by massive swelling resulting in large spherical bacterial cells (Fig. 5C), as has been described for 'cowpea rhizobia' when present in peanut nodules (Chandler 1978;
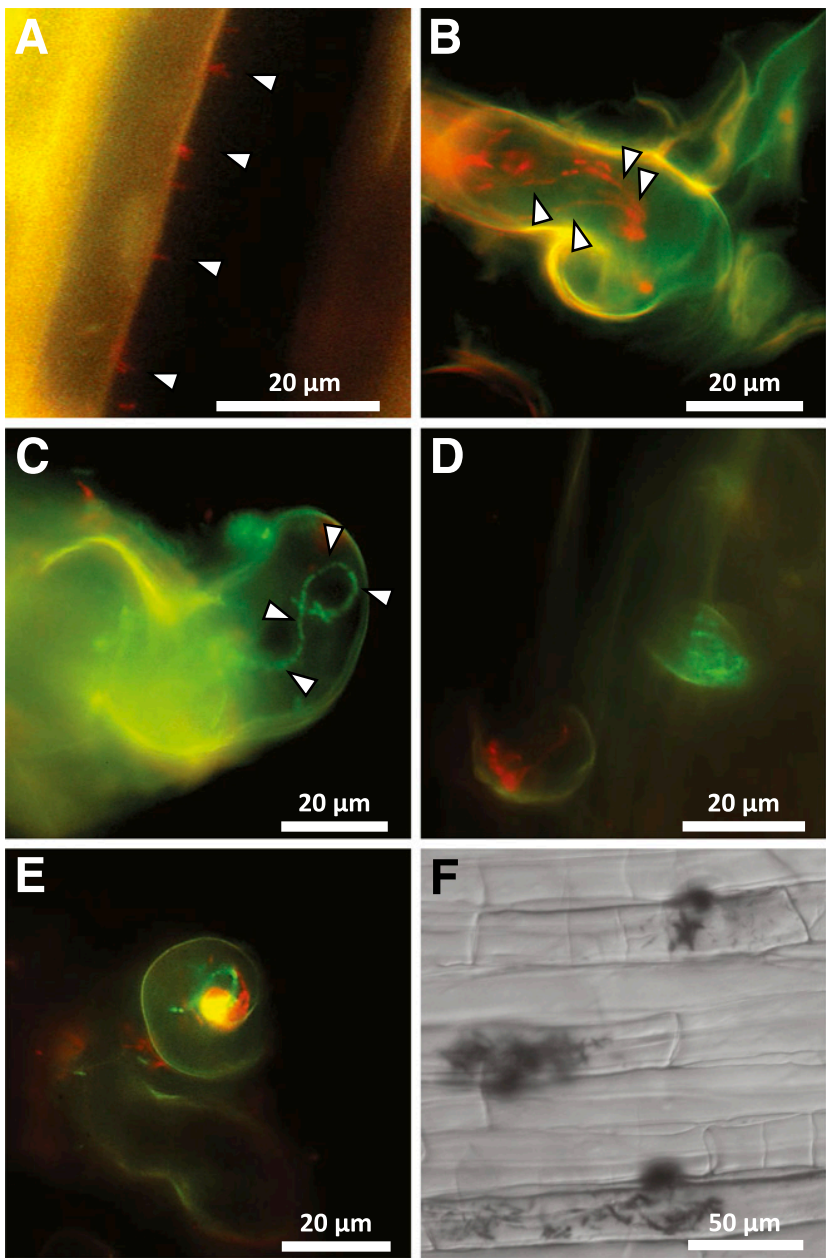

Fig. 2. Visualization of early stages during soybean infection by tagged Bradyrhizobium diazoefficiens strains. A to E, Soybean seedlings were inoculated with B. diazoefficiens strains mChe-1 (red), bjGFP-1 (green), or GusA-1 and were observed by fluorescence or $\mathbf{F}$, differential interference contrast microscopy at 4 days postinoculation (dpi). A, Polar attachment of mChe-1 cells (white arrowheads) to root-hair cells. B, Thin infection threads containing mCherry- or $\mathbf{C}$, green fluorescent protein (GFP)-tagged bacteria (exemplary bacterial cells marked with white arrowheads in both panels) tend to branch very early on during formation, leading to a network. D, After coinoculation of strains mChe-1 and bjGFP-1 (1:1 ratio), individual root-hair cells were infected either by only one strain or $\mathbf{E}$, simultaneously by both strains, via infections threads emerging from a mixed microcolony. F, Visualization of emerging nodule primordia (small, anticlinal dividing plant cells) and associated infection threads by X-gluc staining of GusA-tagged bacteria (dark gray).
Sen et al. 1986). Thus, the concept of host-controlled bacteroid morphology also applies to $B$. diazoefficiens. It remains to be determined whether or not the increased bacteroid size in A. afraspera and peanut nodules is accompanied by additional alterations, such as decreased cell viability or elevated DNA content, which are both characteristic for bacteroids in indeterminate nodules.

\section{Conclusions.}

The plasmids and strains described in this work represent long-desired additions to the molecular toolbox of B. diazoefficiens, the symbiont of the agriculturally important host soybean. For reasons described in the introduction, all marker genes were stably integrated in the chromosome at a uniform locus without discernable phenotypic consequences other than the tag and the tetracycline resistance associated with it. Although expression was driven from a marker gene in single copy, strong transcription and translation was achieved in B. diazoefficiens. Cells tagged with fluorescent or enzymatic marker genes under the control of the Paph or PtuF promoter were readily detected at all stages of symbiosis with different hosts documenting the effectiveness of the constructed strains.
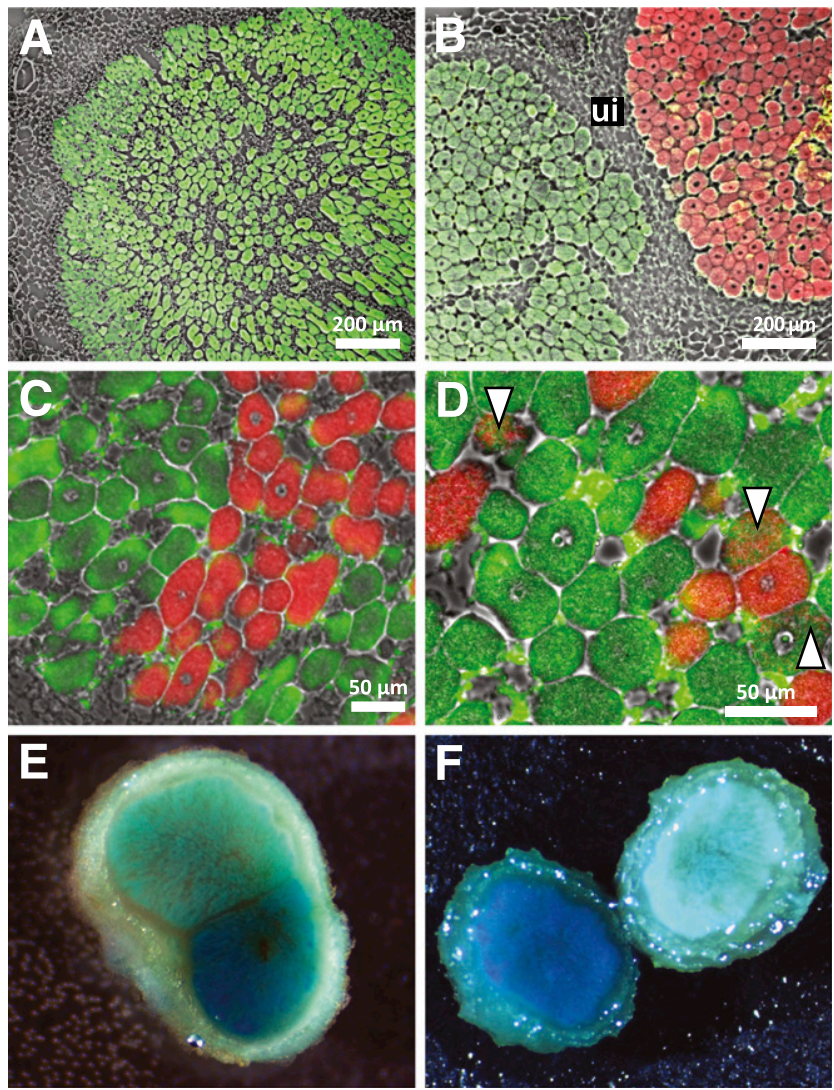

Fig. 3. Nodule colonization patterns observed after mixed infection of soybean seedlings with fluorescently or enzymatically tagged Bradyrhizobium diazoefficiens strains. A to D, Overlays of mCherry- and yellow fluorescent protein (YFP)-derived fluorescence from strains mChe-4 (red) and SYFP-4 (green). Nodules are colonized either by one strain (A, example sYFP2-4-colonized nodule) or by two (B, C, and D). The latter type of nodules comprises 'fused nodules,' seemingly resulting from the fusion of two adjacent emerging nodules, and retain uninfected cells (ui) between zones infected by either of both strains (B) and truly mixed infected nodules in which no uninfected cells were present between zones populated by the two strains (C). In rare cases, individual host cells simultaneously harbored both strains (D, arrowheads). Sections through $\mathbf{E}$, fused and $\mathbf{F}$, clonal nodules, formed upon mixed infection with enzymatically tagged strains GusA-1 (blue) and LacZYA-1 (turquoise) and subsequent staining. 
The constructs made in this study allowed, for the first time, the visualization of autofluorescent $B$. diazoefficiens cells in soybean infection threads, which is particularly challenging given the thin, short, and early branching nature of soybean infection threads compared with other rhizobial systems. Moreover, the

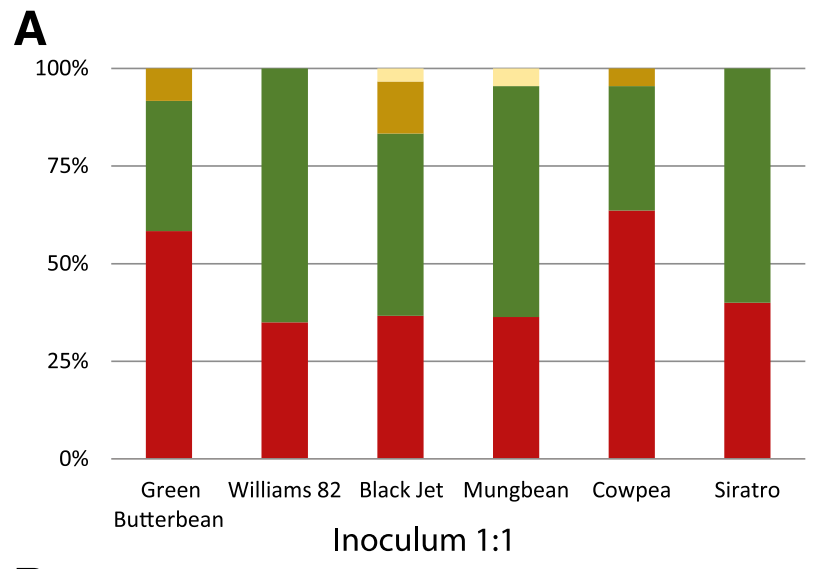

B
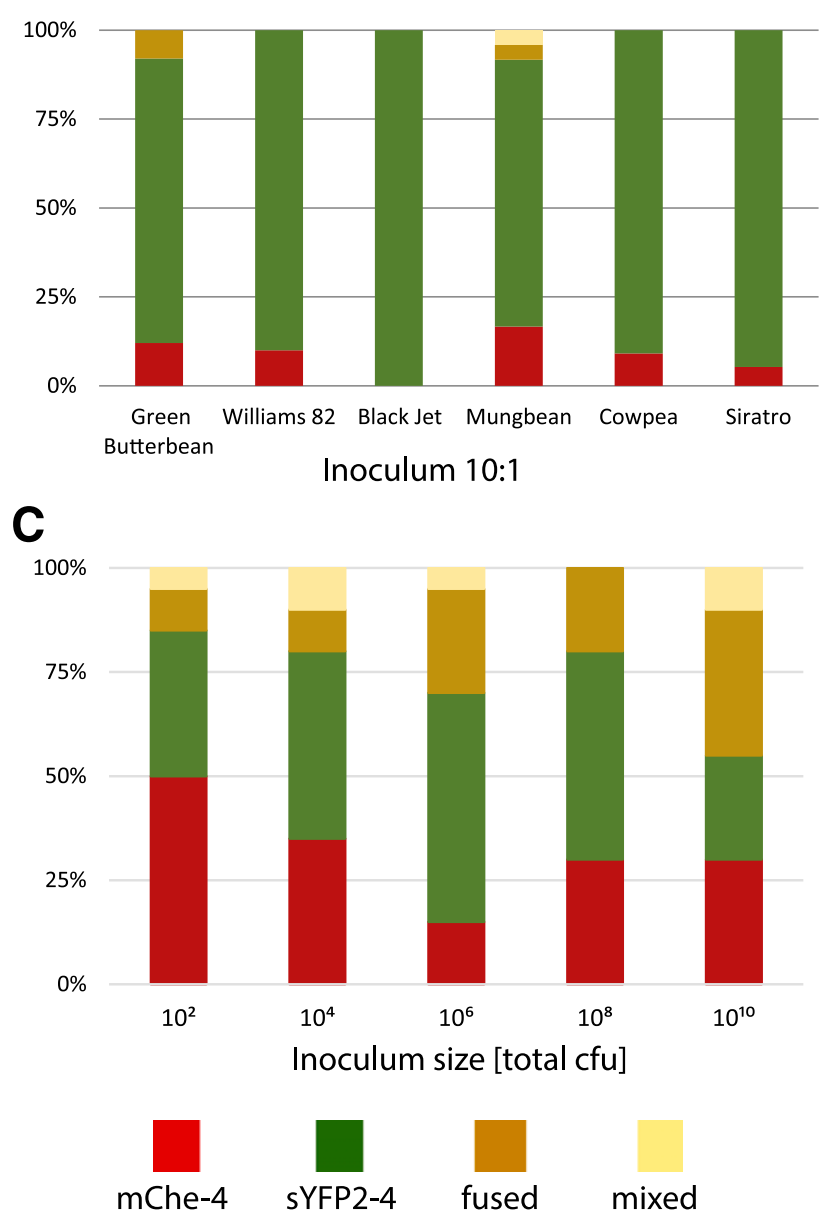

Fig. 4. Use of fluorescently tagged Bradyrhizobium diazoefficiens strains to study nodule occupancy in dependence of host and inoculum composition. Strains sYFP2-4 and mChe-4 were mixed $\mathbf{A}, 1: 1$ or $\mathbf{B}, 10: 1$ at a constant total cell number of $10^{3}$ or 1:1 with $\mathbf{C}$, the specified, increasing total cell numbers and were inoculated on the indicated legumes (A and B) or soybean cv. Green Butterbean (C). From each infection experiment, 10 spherical, well-separated nodules from each of two plants were harvested and nodule occupancy was analyzed by fluorescent microscopy. Shown are percentages of nodules colonized by only one strain or both strains in 'fused' or 'mixed' nodules. genetically tagged strains enabled rapid analysis of nodule occupancy and provided evidence for host-dependent control of B. diazoefficiens bacteroid morphology. These promising results suggest that the tools developed in this study may become particularly useful for the characterization of symbiotically impaired mutants at the level of host infection, colonization, and competitiveness.

Beyond tagging of $B$. diazoefficiens as described here, the plasmids developed herein may also prove useful for other bacteria after replacing the respective $B$. diazoefficiens DNA region with a suitable DNA fragment from the organism of interest. To do so, the dedicated flanking restriction sites can be used to excise the $B$. diazoefficiens DNA and introduce an appropriate piece of DNA (Supplementary Fig. S3). In fact, this strategy has been successfully used for chromosomal insertion of pRJPtuF-mCherry derivatives in three other alphaproteobacterial species, i.e., Rhodopseudomonas palustris TIE-1, M. extorquens AM1, and Sphingomonas melonis FR1 (unpublished), indicating that the genetic tools described offer applications beyond their use in B. diazoefficiens.

\section{MATERIALS AND METHODS}

Bacterial strains, plasmids, media, and growth conditions.

Bacterial strains and plasmids used in this study are listed in Table 1. Escherichia coli strains were routinely grown in lysogeny broth (Miller 1972) at $37^{\circ} \mathrm{C}$. Antibiotics were used at the following concentrations $(\mu \mathrm{g} / \mathrm{ml})$ : ampicillin (200), gentamicin (10), kanamycin (30), tetracycline (10). B. diazoefficiens strains were grown at $30^{\circ} \mathrm{C}$ in PSY medium supplemented with $0.1 \%$ L(+)-arabinose (Mesa et al. 2008) and appropriate antibiotics $(\mu \mathrm{g} / \mathrm{ml})$ : spectinomycin (100), tetracycline (50 in agar, 25 in liquid cultures), chloramphenicol (20; for counterselection of E. coli). Fluorescent cells used for recording excitation and emission spectra were grown in MOPS-buffered minimal medium (Guerinot et al. 1990). Additional details related to strains and plasmids are provided in Supplementary Table S1.

\section{Construction of tagged $B$. diazoefficiens strains.}

All $B$. diazoefficiens strains harboring genetically encoded fluorescent tags were constructed according to a uniform strategy, which is described in detail only for strain GFP-1. Further details about construction of analogous strains are provided in the Supplementary Methods available online. Plasmid pRJ2575 was digested with EcoRI and PstI, releasing a linear DNA fragment that consists of the conjugative vector pSUP202pol4 (Fischer et al. 1993) plus 807 bp of the $B$. diazoefficiens DNA originating from the region downstream of the scoI (blr1131) gene (Bühler et al. 2010). This construct was ligated with a 1,104-bp EcoRI-PstI fragment isolated from plasmid pMG103-npt2-GFP-BisAH-DG1 (Bonaldi et al. 2010), which contains a transcriptional fusion of the promoter of the aphII kanamycin-resistance gene (Paph) to the GFP+ gene (Karunakaran et al. 2005; Scholz et al. 2000). The resulting plasmid pRJPaph-gfp was transformed into E. coli S17-1 $\lambda$ pir, from which it was transferred to $B$. diazoefficiens by biparental conjugation. Upon selection for tetracycline resistance, clones were obtained that harbored the entire plasmid, integrated in the B. diazoefficiens chromosome by homologous recombination downstream of scol. Correct integration at the locus was verified by polymerase chain reaction amplification of DNA regions spanning the recombination site, using primers listed in Supplementary Table S2.

For generation of all other fluorescently tagged $B$. diazoefficiens strains listed in Table 1, the respective plasmids were constructed analogously to pRJPaph-gfp and were integrated into the $B$. diazoefficiens chromosome downstream of scoI. In addition 
to Paph, we also used the promoter associated with the Tu translation elongation factor gene of Methylobacterium extorquens AM1 (PtuF) to drive transcription of selected fluorescent proteinencoding genes (Schada von Borzyskowski et al. 2015). The respective constructs PtuF-eBFP2, PtuF-mTurquoise2, PtuF-sYFP2, and PtuF- $m C h e r r y$ were chromosomally integrated downstream of scol, as described above for the Paph-driven constructs.

Finally, we also constructed two enzymatically tagged B. diazoefficiens strains (lacZYA-1 and gusA-1) containing constitutively expressed lacZYA and gusA genes. They were obtained by integration via homologous recombination of plasmids pRJPaph-lacZYA and pRJPaph-gusA, respectively, into the chromosome of $B$. diazoefficiens wild type, downstream of scoI.

\section{Excitation and emission spectra of tagged \\ B. diazoefficiens cells.}

Fluorescent spectra of cell suspensions were recorded with a Cary Eclipse spectrofluorimeter (Agilent Technologies, Santa Clara, CA, U.S.A.). All measurements were done using the following settings: $5 \mathrm{~nm}$ excitation and emission slits, $30 \mathrm{~nm} / \mathrm{min}$ scan rate, and 1,000 V PMT voltage. Fluorescently tagged bacteria were cultivated in minimal medium, were harvested by centrifugation at early stationary phase, were washed twice in phosphate-buffered saline (PBS), and were adjusted to an optical density at $600 \mathrm{~nm}\left(\mathrm{OD}_{600}\right)=0.75$, except for strain sYFP2-1, which was adjusted to $\mathrm{OD}_{600}=0.15$ because of its high fluorescence. While recording spectra, wavelengths of excitation and emission were offset from the optimal values in order to minimize interference with scattered excitation light. Specifically, spectra were recorded at the following wavelengths (excitation/emission in $\mathrm{nm}$ ): 385/450 (strain eBFP2-1), 435/490 (mTq2-1), 480/520 (bjGFP-1), 505/540 (sYFP2-1), 540/625 (mChe-1), and 550/635 (HcRed-1). Spectra represent means of three recordings (HcRed-1 emission: 5). Spectra were normalized to the respective maximal excitation and emission. To quantify the maximal fluorescent intensity, all strains were excited at their respective maxima, and point measurements at optimal emission wavelengths were recorded. Fluorescent intensity was then normalized to CFU in the measured culture.

\section{Plant inoculation tests.}

Seeds of soybean (Glycine max (L.) Merr.) cv. Williams 82 (provided by D.-N. Rodriguez, CIFA, Las Torres-Tomejil, Seville, Spain), cvs. Black Jet and Green Butterbean, mung bean (Vigna radiata (L.) R. Wilczek), cowpea (Vigna unguiculata (L.) Walp.) cv. Iron \& Clay (Johnny's Selected Seeds, Albion, ME, U.S.A.), siratro (Macroptilium atropurpureum (DC.) Urb.) (provided by W. D. Broughton, University of Geneva, Switzerland), and peanut (Arachis hypogaea L.; TRS Wholesale Co. Ltd. Southall, Middlesex, U.K.) were surface sterilized by immersing in $100 \%$ ethanol for $5 \mathrm{~min}$ and $35 \% \mathrm{H}_{2} \mathrm{O}_{2}$ for
15 min. After intensive washing with sterile water, plant tests were performed as described previously (Göttfert et al. 1990b). Seeds of A. afraspera J. Léonard (provided by E. Giraud, Laboratoire des Symbioses Tropicales et Méditerranéennes, Montpellier, France) were surface-sterilized in concentrated sulfuric acid for $45 \mathrm{~min}$, were washed, were soaked for $24 \mathrm{~h}$ in sterile water, and were processed subsequently, as described above, except that plants were grown under waterlogged conditions. To obtain stem nodules, a previously described procedure was applied (Renier et al. 2011).

\section{Sample preparation and fluorescent microscopy.}

Nodules were routinely harvested 21 days postinoculation (dpi), except for siratro (28 dpi) and peanuts (35 dpi), were fixed in $4 \%$ formalin in PBS $\left(1.9 \mathrm{mM} \mathrm{NaH} \mathrm{PO}_{4}, 8.1 \mathrm{mM}\right.$ $\mathrm{Na}_{2} \mathrm{HPO}_{4}, 140 \mathrm{mM} \mathrm{NaCl}$ ) at $4{ }^{\circ} \mathrm{C}$ overnight, were washed twice in PBS supplemented with $50 \mathrm{mM} \mathrm{NH}_{4} \mathrm{Cl}$, were dehydrated in $20 \%$ (wt/vol) sucrose in PBS at $4{ }^{\circ} \mathrm{C}$ for $24 \mathrm{~h}$, were briefly rinsed in $80 \%$ ethanol, and were embedded in TissueTek O.C.T. Compound (Sakura Finetek Europe B.V., Alphen aan den Rjin, The Netherlands) before shock-freezing in liquid nitrogen. Semithin sections of $10 \mu \mathrm{m}$ were prepared by using a Microtome Cryostat HM525 (Microm International GmbH, Walldorf, Germany) and were mounted on glass slides. Sections on glass slides were washed in PBS, air dried, and mounted in 50\% glycerol, before microscopic examination. To acquire high-quality micrographs, sections were mounted with the antifade mounting resin citifluor AF1 (Citifluor Ltd., London). Epifluorescence microscopy was performed using Zeiss filter sets: 13 (GFP/sYFP2) or 15 or 43 (mCherry). At least three nonconsecutive sections per nodule were examined to evaluate the bacterial colonization.

For observation of root-hair attachment and infection threads, roots were harvested $4 \mathrm{dpi}$, were rinsed in water to remove vermiculite particles, and were washed as described elsewhere (Bulgarelli et al. 2012). Epidermal cell layers of roots were peeled off using a blunt razor blade and were mounted on glass slides in $50 \%$ glycerol.

High-resolution images of bacteroids were recorded using confocal microscopy. For soybean and peanut bacteroids, a Leica SP2-FCS point laser scanning confocal microscope (Leica Microsystems AG, Heerbrugg, Switzerland) was used. mCherry was excited with a HeNe laser at $594 \mathrm{~nm}$. Fluorescence emission was recorded between 600 and $648 \mathrm{~nm}$. For enhanced contrast, plant tissue autofluorescence was excited at $514 \mathrm{~nm}$ with an argon laser and emission was recorded between 520 and $560 \mathrm{~nm}$. Bacteroids in A. afraspera nodules were imaged using a Visitron spinning disc confocal system (Visitron Systems GmbH, Puchheim, Germany) on a Zeiss 200M microscope. Bacteroids expressing mCherry were excited at $561 \mathrm{~nm}$ and emission was recorded with an ET 605/52M filter (Chroma Technology, Bellows Falls, VT, U.S.A.). Autofluorescence of plant tissue was excited at $488 \mathrm{~nm}$ and was recorded with an ET 525/50M filter.
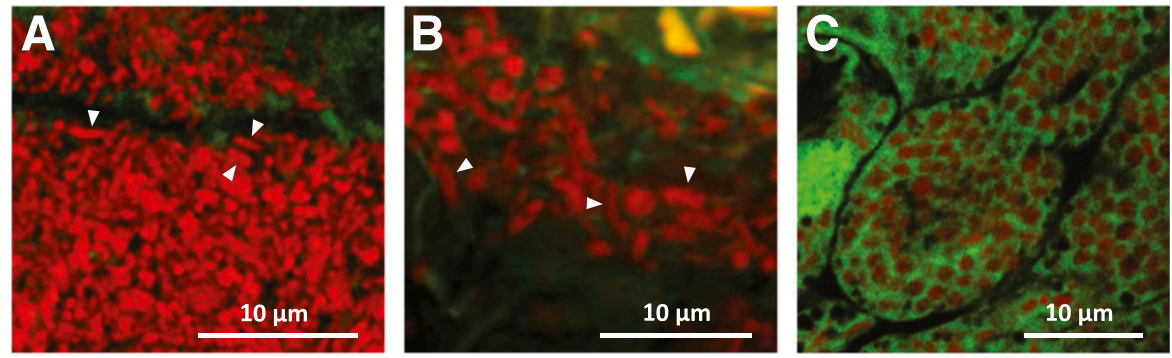

Fig. 5. Host-dependent morphology and size of Bradyrhizobium diazoefficiens bacteroids. A, Confocal micrographs of mCherry-tagged B. diazoefficiens mChe-1 bacteroids present in infected cells of soybean, B, Aeschynomene afraspera, or $\mathbf{C}$, peanut root nodules. Bacteroids are rod shaped in soybean, enlarged to various extents, and hence, irregularly shaped in A. afraspera or very large and spherical in peanut. To highlight the difference in size distinct bacteroids are labeled with white arrowheads in panels $\mathrm{A}$ and $\mathrm{B}$. 


\section{Staining for GusA and LacZ activity.}

For observations of early infection stages, roots of $B$. diazoefficiens GusA-1-inoculated soybean plants were harvested 4 dpi and were washed as described above. GusA activity in bacteria present in infection threads was visualized according to a modified staining protocol described previously (Wilson et al. 1995). In short, roots were placed in staining solution $(19.5 \mathrm{mM}$ $\mathrm{NaH}_{2} \mathrm{PO}_{4}, 30.5 \mathrm{mM} \mathrm{Na}_{2} \mathrm{HPO}_{4}, 1 \mathrm{mM}$ EDTA, $1 \mathrm{mM} \mathrm{K}_{3}\left[\mathrm{Fe}(\mathrm{CN})_{6}\right]$, $0.1 \%$ Triton X-100, $0.1 \%$ sarkosyl) and $200 \mu$ g of X-gluc per milliliter, were vacuum-treated for $20 \mathrm{~min}$, and were incubated $12 \mathrm{~h}$ at $37^{\circ} \mathrm{C}$. Subsequently, roots were cleared with chloral hydrate (Hayashi et al. 2005), were sliced longitudinally, and were observed by differential interference contrast light microscopy.

For simultaneous detection of GusA and LacZ activity in mixed infected soybean nodules, nodules were harvested $23 \mathrm{dpi}$, were detached from roots, were washed in PBS amended with $0.02 \%$ Silvet L-77, were cut in half, were washed again briefly in PBS, and were placed in the staining solution described above. X-gluc and green- $\beta$-D-gal (both purchased from Biosynth, Staad, Switzerland) were added to a concentration of $100 \mu \mathrm{g} / \mathrm{ml}$ each, and nodules were vacuum-treated for $30 \mathrm{~min}$, followed by incubation at $28^{\circ} \mathrm{C}$ for $4 \mathrm{~h}$ before they were observed under a stereo microscope. Endogenous plant-derived $\beta$-galactosidase activity was detected only after prolonged incubation, i.e., it did not interfere with LacZ-derived activity and there was no need for its inhibition by preceding glutaraldehyde fixation.

\section{ACKNOWLEDGMENTS}

We thank T. Erb and R. Weishaupt for providing plasmid pTE-HcRed, E. Giraud for plasmid pMG103-npt2-GFP-BisAH-DG1, M. Schlumberger for technical assistance in confocal microscopy, and D. Nombre RodríguezNavarro and W. Broughton for legume seeds. H. Hennecke is greatly acknowledged for critically reading the manuscript and constructive comments. This work was supported by grants from ETH Zürich and from the Swiss National Foundation for Scientific Research.

\section{LITERATURE CITED}

Avarez-Morales, A., Betancourt-Alvarez, M., Kaluza, K., and Hennecke, H. 1986. Activation of the Bradyrhizobium japonicum nifH and nifDK operons is dependent on promoter-upstream DNA sequences. Nucleic Acids Res. 14:4207-4227.

Bhatia, R., Dogra, R. C., and Sharma, P. K. 2002. Construction of green fluorescent protein (GFP)-marked strains of Bradyrhizobium for ecological studies. J. Appl. Microbiol. 93:835-839.

Bonaldi, K., Gargani, D., Prin, Y., Fardoux, J., Gully, D., Nouwen, N., Goormachtig, S., and Giraud, E. 2011. Nodulation of Aeschynomene afraspera and $A$. indica by photosynthetic Bradyrhizobium sp. strain ORS285: The nod-dependent versus the nod-independent symbiotic interaction. Mol. Plant Microbe Interact. 24:1359-1371.

Bonaldi, K., Gherbi, H., Franche, C., Bastien, G., Fardoux, J., Barker, D., Giraud, E., and Cartieaux, F. 2010. The Nod factor-independent symbiotic signaling pathway: Development of Agrobacterium rhizogenes-mediated transformation for the legume Aeschynomene indica. Mol. Plant Microbe Interact. 23:1537-1544.

Bühler, D., Rossmann, R., Landolt, S., Balsiger, S., Fischer, H. M., and Hennecke, H. 2010. Disparate pathways for the biogenesis of cytochrome oxidases in Bradyrhizobium japonicum. J. Biol. Chem. 285:15704-15713.

Bulgarelli, D., Rott, M., Schlaeppi, K., Ver Loren van Themaat, E., Ahmadinejad, N., Assenza, F., Rauf, P., Huettel, B., Reinhardt, R., Schmelzer, E., Peplies, J., Gloeckner, F. O., Amann, R., Eickhorst, T., and Schulze-Lefert, P. 2012. Revealing structure and assembly cues for Arabidopsis root-inhabiting bacterial microbiota. Nature 488:91-95.

Calvert, H. E., Pence, M. K., Pierce, M., Malik, N. S. A., and Bauer, W. D. 1984. Anatomical analysis of the development and distribution of Rhizobium infections in soybean roots. Can. J. Bot. 62:23752384.

Chandler, M. R. 1978. Some observations on infection of Arachis hypogaea L. by Rhizobium. J. Exp. Bot. 29:749-755.
Choi, K. H., Gaynor, J. B., White, K. G., Lopez, C., Bosio, C. M., KarkhoffSchweizer, R. R., and Schweizer, H. P. 2005. A Tn7-based broad-range bacterial cloning and expression system. Nat. Methods 2:443-448.

Day, R. N., and Davidson, M. W. 2009. The fluorescent protein palette: Tools for cellular imaging. Chem. Soc. Rev. 38:2887-2921.

de Lorenzo, V., Cases, I., Herrero, M., and Timmis, K. N. 1993. Early and late responses of TOL promoters to pathway inducers: identification of postexponential promoters in Pseudomonas putida with lacZ-tet bicistronic reporters. J. Bacteriol. 175:6902-6907.

Delamuta, J. R., Ribeiro, R. A., Ormeño-Orrillo, E., Melo, I. S., MartínezRomero, E., and Hungria, M. 2013. Polyphasic evidence supporting the reclassification of Bradyrhizobium japonicum group Ia strains as Bradyrhizobium diazoefficiens sp. nov. Int. J. Syst. Evol. Microbiol. 63:3342-3351.

Downie, J. A. 2014. Legume nodulation. Curr. Biol. 24: R184-R190.

Fischer, H. M., Babst, M., Kaspar, T., Acuña, G., Arigoni, F., and Hennecke, H. 1993. One member of a gro-ESL-like chaperonin multigene family in Bradyrhizobium japonicum is co-regulated with symbiotic nitrogen fixation genes. EMBO J. 12:2901-2912.

Gage, D. J. 2002. Analysis of infection thread development using Gfpand DsRed-expressing Sinorhizobium meliloti. J. Bacteriol. 184: 7042-7046.

Gage, D. J., Bobo, T., and Long, S. R. 1996. Use of green fluorescent protein to visualize the early events of symbiosis between Rhizobium meliloti and alfalfa (Medicago sativa). J. Bacteriol. 178:7159-7166.

Goedhart, J., von Stetten, D., Noirclerc-Savoye, M., Lelimousin, M., Joosen, L., Hink, M. A., van Weeren, L., Gadella, T. W., Jr., and Royant, A. 2012. Structure-guided evolution of cyan fluorescent proteins towards a quantum yield of $93 \%$. Nat. Commun. 3:751.

Göttfert, M., Grob, P., and Hennecke, H. 1990a. Proposed regulatory pathway encoded by the nodV and nodW genes, determinants of host specificity in Bradyrhizobium japonicum. Proc. Natl. Acad. Sci. U.S.A. 87:2680-2684.

Göttfert, M., Hitz, S., and Hennecke, H. 1990b. Identification of nodS and nodU, two inducible genes inserted between the Bradyrhizobium japonicum nodYABC and nodIJ genes. Mol. Plant Microbe Interact. 3: 308-316.

Grote, A., Hiller, K., Scheer, M., Münch, R., Nörtemann, B., Hempel, D. C., and Jahn, D. 2005. JCat: A novel tool to adapt codon usage of a target gene to its potential expression host. Nucleic Acids Res. 33:W526W531.

Guerinot, M. L., Meidl, E. J., and Plessner, O. 1990. Citrate as a siderophore in Bradyrhizobium japonicum. J. Bacteriol. 172:3298-3303.

Gurskaya, N. G., Fradkov, A. F., Terskikh, A., Matz, M. V., Labas, Y. A., Martynov, V. I., Yanushevich, Y. G., Lukyanov, K. A., and Lukyanov, S. A. 2001. GFP-like chromoproteins as a source of far-red fluorescent proteins. FEBS Lett. 507:16-20.

Hauser, F., Pessi, G., Friberg, M., Weber, C., Rusca, N., Lindemann, A., Fischer, H. M., and Hennecke, H. 2007. Dissection of the Bradyrhizobium japonicum NifA $+\sigma^{54}$ regulon, and identification of a ferredoxin gene $(f d x N)$ for symbiotic nitrogen fixation. Mol. Genet. Genomics 278: 255-271.

Hayashi, M., Shiro, S., Kanamori, H., Mori-Hosokawa, S., SasakiYamagata, H., Sayama, T., Nishioka, M., Takahashi, M., Ishimoto, M., Katayose, Y., Kaga, A., Harada, K., Kouchi, H., Saeki, Y., and Umehara, Y. 2014. A thaumatin-like protein, Rj4, controls nodule symbiotic specificity in soybean. Plant Cell Physiol. 55:1679-1689.

Hayashi, M., Tansengco, M. L., Suganuma, N., Szczyglowski, K., Krusell, L., Ott, T., and Udvardi, M. 2005. Methods for studying nodule development and function. Pages 53-82. in: Lotus japonicus handbook. A. J. Márquez, ed. Springer, Dordrecht, The Netherlands.

Karunakaran, R., Mauchline, T. H., Hosie, A. H., and Poole, P. S. 2005. A family of promoter probe vectors incorporating autofluorescent and chromogenic reporter proteins for studying gene expression in gramnegative bacteria. Microbiology 151:3249-3256.

Koch, B., Jensen, L. E., and Nybroe, O. 2001. A panel of Tn7-based vectors for insertion of the $g f p$ marker gene or for delivery of cloned DNA into gram-negative bacteria at a neutral chromosomal site. J. Microbiol. Methods 45:187-195.

Kondorosi, E., Mergaert, P., and Kereszt, A. 2013. A paradigm for endosymbiotic life: Cell differentiation of Rhizobium bacteria provoked by host plant factors. Annu. Rev. Microbiol. 67:611-628.

Matthysse, A. G., Stretton, S., Dandie, C., McClure, N. C., and Goodman, A. E. 1996. Construction of GFP vectors for use in gram-negative bacteria other than Escherichia coli. FEMS Microbiol. Lett. 145:87-94.

Mergaert, P., Uchiumi, T., Alunni, B., Evanno, G., Cheron, A., Catrice, O., Mausset, A. E., Barloy-Hubler, F., Galibert, F., Kondorosi, A., and Kondorosi, E. 2006. Eukaryotic control on bacterial cell cycle and 
differentiation in the Rhizobium-legume symbiosis. Proc. Natl. Acad. Sci. U.S.A. 103:5230-5235.

Mesa, S., Hauser, F., Friberg, M., Malaguti, E., Fischer, H. M., and Hennecke, H. 2008. Comprehensive assessment of the regulons controlled by the FixLJ-FixK ${ }_{2}-$FixK$_{1}$ cascade in Bradyrhizobium japonicum. J. Bacteriol. 190:6568-6579.

Miller, J. H. 1972. Experiments in molecular genetics. Cold Spring Harbor Laboratory Press, Cold Spring Harbor, NY, U.S.A.

Mylle, E., Codreanu, M. C., Boruc, J., and Russinova, E. 2013. Emission spectra profiling of fluorescent proteins in living plant cells. Plant Methods 9:10.

Noisangiam, R., Teamtisong, K., Tittabutr, P., Boonkerd, N., Toshiki, U., Minamisawa, K., and Teaumroong, N. 2012. Genetic diversity, symbiotic evolution, and proposed infection process of Bradyrhizobium strains isolated from root nodules of Aeschynomene americana $\mathrm{L}$. in Thailand. Appl. Environ. Microbiol. 78:6236-6250.

Okubo, T., Fukushima, S., Itakura, M., Oshima, K., Longtonglang, A., Teaumroong, N., Mitsui, H., Hattori, M., Hattori, R., Hattori, T., and Minamisawa, K. 2013. Genome analysis suggests that the soil oligotrophic bacterium Agromonas oligotrophica (Bradyrhizobium oligotrophicum) is a nitrogen-fixing symbiont of Aeschynomene indica. Appl. Environ. Microbiol. 79:2542-2551.

Oldroyd, G. E., and Downie, J. A. 2008. Coordinating nodule morphogenesis with rhizobial infection in legumes. Annu. Rev. Plant Biol. 59:519-546.

Oldroyd, G. E., Murray, J. D., Poole, P. S., and Downie, J. A. 2011. The rules of engagement in the legume-rhizobial symbiosis. Annu. Rev. Genet. 45:119-144.

Oono, R., Schmitt, I., Sprent, J. I., and Denison, R. F. 2010. Multiple evolutionary origins of legume traits leading to extreme rhizobial differentiation. New Phytol. 187:508-520.

Pessi, G., Ahrens, C. H., Rehrauer, H., Lindemann, A., Hauser, F., Fischer, H. M., and Hennecke, H. 2007. Genome-wide transcript analysis of Bradyrhizobium japonicum bacteroids in soybean root nodules. Mol. Plant Microbe Interact. 20:1353-1363.

Pobigaylo, N., Szymczak, S., Nattkemper, T. W., and Becker, A. 2008. Identification of genes relevant to symbiosis and competitiveness in Sinorhizobium meliloti using signature-tagged mutants. Mol. Plant Microbe Interact. 21:219-231.

Pueppke, S. G. 1983. Rhizobium infection threads in root hairs of Glycine $\max$ (L.) Merr., Glycine soja Sieb. \& Zucc, and Vigna unguiculata (L.). Can. J. Microbiol. 29:69-76.
Ramseier, T. M., and Göttfert, M. 1991. Codon usage and G + C content in Bradyrhizobium japonicum genes are not uniform. Arch. Microbiol. 156: 270-276.

Regensburger, B., and Hennecke, H. 1983. RNA polymerase from Rhizobium japonicum. Arch. Microbiol. 135:103-109.

Renier, A., Maillet, F., Fardoux, J., Poinsot, V., Giraud, E., and Nouwen, N. 2011. Photosynthetic Bradyrhizobium sp. strain ORS285 synthesizes 2-O-methylfucosylated lipochitooligosaccharides for nod gene-dependent interaction with Aeschynomene plants. Mol. Plant Microbe Interact. 24: 1440-1447.

Schada von Borzyskowski, L., Remus-Emsermann, M., Weishaupt, R., Vorholt, J. A., and Erb, T. J. 2015. A set of versatile brick vectors and promoters for the assembly, expression, and integration of synthetic operons in Methylobacterium extorquens AM1 and other alphaproteobacteria. ACS Synth Biol 4:430-443.

Scholz, O., Thiel, A., Hillen, W., and Niederweis, M. 2000. Quantitative analysis of gene expression with an improved green fluorescent protein. p6. Eur. J. Biochem. 267:1565-1570.

Sen, D., Weaver, R. W., and Bal, A. K. 1986. Structure and organization of effective peanut and cowpea root nodules induced by rhizobial strain 32H1. J. Exp. Bot. 37:356-363.

Simms, E. L., Taylor, D. L., Povich, J., Shefferson, R. P., Sachs, J. L., Urbina, M., and Tausczik, Y. 2006. An empirical test of partner choice mechanisms in a wild legume-rhizobium interaction. Proc. R. Soc. B. 273:77-81.

Stuurman, N., Pacios Bras, C., Schlaman, H. R., Wijfjes, A. H., Bloemberg, G., and Spaink, H. P. 2000. Use of green fluorescent protein color variants expressed on stable broad-host-range vectors to visualize rhizobia interacting with plants. Mol. Plant Microbe Interact. 13:1163-1169.

Turgeon, B. G., and Bauer, W. D. 1985. Ultrastructure of infection-thread development during the infection of soybean by Rhizobium japonicum. Planta 163:328-349.

Wilson, K. J., Sessitsch, A., Corbo, J. C., Giller, K. E., Akkermans, A. D., and Jefferson, R. A. 1995. $\beta$-Glucuronidase (GUS) transposons for ecological and genetic studies of rhizobia and other gram-negative bacteria. Microbiology 141:1691-1705.

\section{AUTHOR-RECOMMENDED INTERNET RESOURCE}

Addgene plasmid repository: www.addgene.org 